\title{
Finite Element Analysis of Tilting of Metal in Bar Rolled with Three-roll Mill
}

\author{
Motoo ASAKAWA, ${ }^{1)}$ Kengo KOBAYASHI, ${ }^{2)}$ Yuki KATAYAMA, ${ }^{2)}$ Daisuke SHIOGA ${ }^{1)}$ and Jun YANAGIMOTO ${ }^{3)}$ \\ 1) Department of Science and Engineering, Waseda University, 3-4-1 Ookubo, Shinjuku, Tokyo 169-8555 Japan. Email: \\ asakawa@waseda.jp \\ Tokyo 169-8555 Japan. \\ 2) Graduate School of Science and Engineering, Waseda University, 3-4-1 Ookubo, Shinjuku, \\ 3) Institute of Industrial Science, The University of Tokyo, 4-6-1 Komaba, Meguro, Tokyo 153-
} 8505 Japan.

(Received on July 21, 2005; accepted on December 28, 2005)

\begin{abstract}
Rolled metal bars and rods often exhibit tilting, which is rotation of the material around the pass line under rolling. Tilting may result in a defective shape. High dimensional accuracy is demanded in 3-roll rolling because 3-roll rolling is used for finishing passes. Therefore, the design of calibers and a rolling sequence that can reduce tilting is important from industrial aspects. In this study, three-dimensional FE analysis is applied to the prediction of metal tilting under 3-roll rolling in an oval-round pass and a hexagon-oval pass. The difference in the characteristics of tilting according to the contact contour between metal and the roll is investigated by classifying the characteristics of tilting into two modes, with the aim of clarifying the threshold for the occurrence of overturning and restoration resulting from tilting. Predicted results agree well with the experimental measurements of tilting, and the validity of method for analyzing tilting is confirmed. Moreover, it is found that the mechanism of tilting differs according to the contact contour. In an oval-round pass that is of a convex contact type, the bar tilt angle is difficult to decrease once tilting has occurred. In a hexagon-oval pass that is of a concave contact type, although the bar tilt angle tends to increase when the entrance cross section is constrained strongly, the bar tilt angle tends to decrease after constraint becomes relatively small.
\end{abstract}

KEY WORDS: bar rolling; wire rod rolling; tilt; overturning; restoration; three-roll mill; finite element method (FEM).

\section{Introduction}

Rolled metal bars and rods often exhibit tilting due to the small width to height ratio of the bar and rod under rolling. Tilting is the rotation of the metal around the pass line under rolling, as is shown schematically in Fig. 1. Slight tilting may result in wrinkling and overfilling of the rolled product, and severe tilting may result in miss-rolling of the bar or rod. Therefore the design of calibers and a rolling sequence which can reduce tilting is important from industrial aspects.

For 2-roll rolling, Ohno, ${ }^{1)}$ Shternov $^{2)}$ and Asakawa ${ }^{3)}$ ana-

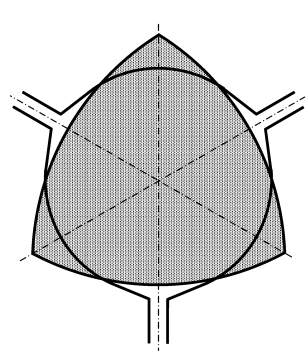

Regular position

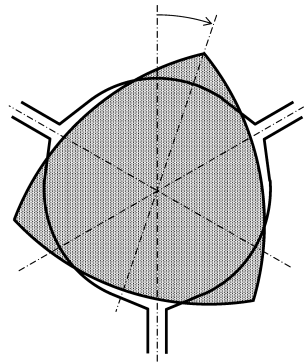

Tilting
Fig. 1. Tilting of workpiece under bar and rod rolling. lyzed tilting by elemental methods. In recent years, threedimensional FE analysis has been applied to the prediction of the tilting of metals under rolling. ${ }^{4-6)}$ However, there has been no research on tilting under 3 -roll rolling. High dimensional accuracy is demanded in 3-roll rolling because it is used mainly for finishing passes. Therefore tilting becomes a major problem in 3-roll rolling.

In this study, three-dimensional FE analysis is applied to the prediction of the tilting of metal under 3-roll rolling in a hexagon-oval pass and an oval-round pass. The validity of analytical results is examined by comparing the results of numerical analyses and experimental measurements. Moreover, the difference in the characteristics of tilting according to the contact contour is investigated, with the aim of clarifying the threshold for the occurrence of overturning and restoration resulting from tilting. Overturning means increase in tilt angle under rolling, and restoration means decrease in tilt angle under rolling.

\section{Analytical Method}

\subsection{Velocity and Force Vectors on the Plane of Sym-} metry

Tilting is analyzed using the FE simulator, 'CORMILL System', which is based on Lagrange multiplier three-di- 


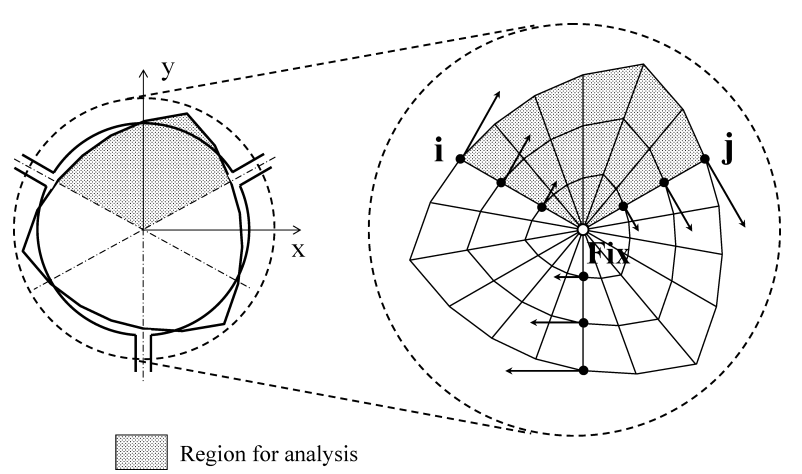

Fig. 2. Distribution of cross-sectional velocity component with twisting of workpiece.

mensional rigid-plastic FE analysis. ${ }^{7-9)}$ It is applicable to steady-state metal flow in sheet, ${ }^{10-12)}$ bar, wire-rod ${ }^{12,13)}$ and shape-rolling ${ }^{12,14)}$ processes.

Usually, the analytic region of 3-roll rolling is one-sixth of the whole deformation zone because of the symmetry of deformation and for the reduction of computational time. However, when tilting occurs, the analytical region must be changed from one-sixth to one-third because cross-sectional metal flow in the hoop direction becomes evident. Because tilting, such as overturning and restoration, results from the cross-sectional metal flow in the hoop direction, we should adopt the periodic boundary condition at the nodal points on the plane of symmetry. Figure 2 shows the plastic flow in the cross-section perpendicular to the rolling direction. One-third of the cross section is analyzed under the velocity boundary condition shown as Eq. (1) and the force boundary condition shown as Eq. (2), which correspond to the periodic boundary condition, in the plane of symmetry.

For node $i$ and node $j$ at periodic positions on the symmetric plane, nodal velocity $\dot{u}$ is written as

$$
\begin{aligned}
& \dot{u}_{x}^{\langle j\rangle}=-\frac{1}{2} \dot{u}_{x}^{\langle i\rangle}+\frac{\sqrt{3}}{2} \dot{u}_{y}^{\langle i\rangle} \\
& \dot{u}_{y}^{\langle j\rangle}=-\frac{\sqrt{3}}{2} \dot{u}_{x}^{\langle i\rangle}-\frac{1}{2} \dot{u}_{y}^{\langle i\rangle} \\
& \dot{u}_{z}^{\langle j\rangle}=\dot{u}_{z}^{\langle i\rangle}
\end{aligned}
$$

Nodal force $F$ is written as

$$
\begin{aligned}
& -\frac{1}{2} F_{x}^{\langle i\rangle}+\frac{\sqrt{3}}{2} F_{y}^{\langle i\rangle}+F_{x}^{\langle j\rangle}=0 \\
& -\frac{\sqrt{3}}{2} F_{x}^{\langle i\rangle}-\frac{1}{2} F_{y}^{\langle i\rangle}+F_{y}^{\langle j\rangle}=0 \\
& F_{z}^{\langle j\rangle}+F_{z}^{\langle i\rangle}=0
\end{aligned}
$$

where $x, y$, and $z$ are the width (lateral), height and rolling directions, respectively.

The velocity perturbation that is obtained by the Newton-Raphson method should also obey the periodic symmetrical condition described by Eq. (3).

$$
\begin{aligned}
& \Delta \dot{u}_{x}^{\langle j\rangle}=-\frac{1}{2} \Delta \dot{u}_{x}^{\langle i\rangle}+\frac{\sqrt{3}}{2} \Delta \dot{u}_{y}^{\langle i\rangle} \\
& \Delta \dot{u}_{y}^{\langle j\rangle}=-\frac{\sqrt{3}}{2} \Delta \dot{u}_{x}^{\langle i\rangle}-\frac{1}{2} \Delta \dot{u}_{x}^{\langle i\rangle} \\
& \Delta \dot{u}_{z}^{\langle j\rangle}=\Delta \dot{u}_{z}^{\langle i\rangle}
\end{aligned}
$$

The velocities at the entrance and exit of the deformation zone are expressed by Eq. (4).

$$
\begin{aligned}
& \dot{u}_{x}=0 \\
& \dot{u}_{y}=0 \ldots \ldots \ldots \ldots \ldots \\
& \dot{u}_{z}=\text { (uniform) } \\
& \dot{u}_{x}=-r \omega \sin \varphi \\
& \dot{u}_{y}=r \omega \cos \varphi \ldots \ldots \\
& \dot{u}_{z}=\text { (uniform) }
\end{aligned}
$$$$
\text { Entrance: } \quad \dot{u}_{y}=0 \text {. }
$$

Exit:

Here, $\omega$ is the angular velocity at the exit, which is obtained by FE analysis as follows.

$$
\begin{aligned}
& \omega=(\text { uniform }, \text { unknown }) \\
& r=\sqrt{x^{2}+y^{2}} \\
& \varphi=\tan ^{-1} \frac{y}{x}
\end{aligned}
$$

$x$ and $y$ are the lateral and horizontal coordinates of nodal points. Equations (4) and (5) show that the bar is fixed by the guide at the entrance, and rotational metal flow caused by tilting is allowed at the exit of the deformation zone.

\subsection{Contact Boundary Condition between Rod and Rolls}

As for the nodal points in contact with rolls, the condition $\dot{\mathbf{u}} \cdot \mathbf{n}=0$ should be satisfied to ensure plastic flow of the bar in the tangential plane of work rolls. The simplest modeling requires the implementation of one work roll, which is one-third of the whole roll arrangement, in the analytical region of one-third of the whole bar under rolling. However, due to rotational metal flow, nodal points on the periodic plane may flow to a position of interference with another roll. Since the implementation of another roll in numerical analysis is not always favorable to ensure short computational time and precise numerical results, the following algorithms are added. In contact analysis, the positions of candidate nodal points are monitored to determine whether they interfere with another roll (side roll, for instance). When a node of the material interferes with the side roll, the analytic region is changed so that the node is located on the boundary line, as is shown in Fig. 3. When node $A$ is not located on the boundary line, node $A$ interferes with the side roll. When node $A$ is located on the boundary line, node $A$ can be prevented from interfering with the side roll so that node $\mathrm{B}$ is in contact with the upper roll. 


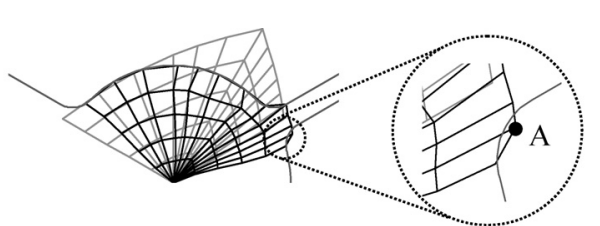

Material interferes in the side roll.

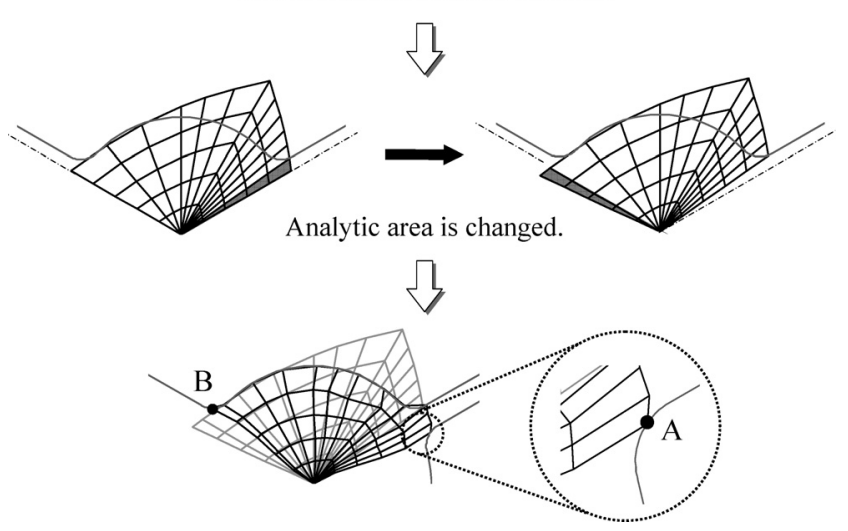

Fig. 3. Outline of the algorithm to prevent interference of the side roll.

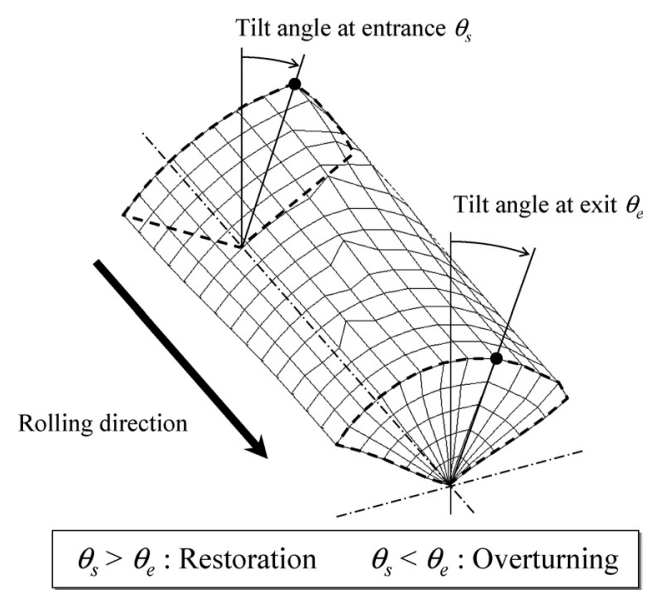

Fig. 4. Measuring points for evaluation of restoration or overturning.

\section{Mode of Tilting and Evaluation Method of Tilting}

Tilting of a bar under rolling can be classified into two modes. Mode 1 tilting takes place even though the position of the entrance cross section is constrained strongly by the guide or mechanical stand. This mode results from steadystate twisting deformation of the roll bite, and this mode can be directly analyzed by steady-state FE analysis. By comparing the tilt angle at the entrance, $\theta_{\mathrm{s}}$, with that at the exit, $\theta_{e}$, restoration or overturning is identified, as is shown in Fig. 4.

Mode 2 tilting takes place in the bar before rolling, when constraint from the guide or mechanical stand is relatively small. This mode results from the moment arising in the plastic deformation zone near the roll bite, and tilting at the entrance changes unsteadily. Accordingly, twisting moment $M_{\text {Ent }}$, shown in Fig. 5, is calculated by FE analysis.

The following parameters control the rolling characteristics in bar and rod rolling.

- Dimensions of the stock and roll profile: Shape of cross section, contact contour, and distribution of load

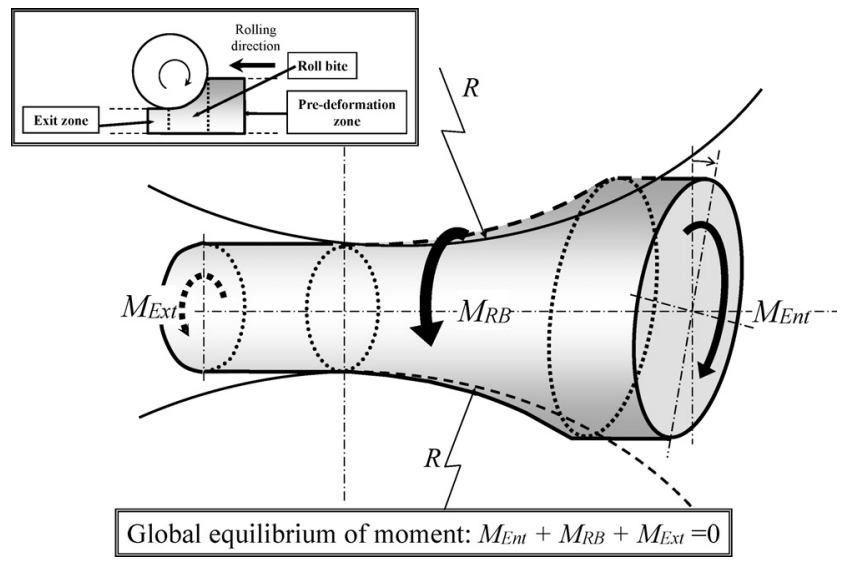

Fig. 5. Moment acting on metal under rolling.

- Roll diameter: Maximum projected contact length, absolute value of load, and velocity distribution

- Temperature of metal: Absolute value of load, contact contour

- Front/Back tension: Shape of cross section, contact contour, and absolute value of load

There are four parameters that vary with rolling characteristics: shape of cross section, contact contour, distribution of load, and absolute value of load. By substituting the data obtained from FE analysis for the four parameters, the following parameters can be obtained.

- Contact contour, distribution of load: twisting moment acting at entrance of bar

- Shape of cross section: polar moment of inertia of area

- Absolute value of load: Average flow stress of metal In order to remove the effects of rolling force and geometry from the torsion rigidity, twisting moment $M_{\mathrm{Ent}}$ is divided by the polar moment of inertia at the entrance, $I_{z}$, and the average flow stress of the roll bite, $\bar{\sigma}_{\text {ave }}$, as $/ M_{\mathrm{Em}} /\left(I_{z} \cdot \bar{\sigma}_{\text {ave }}\right)$ to express the torsion angle per unit 'representative' length. The maximum projected contact length could be assumed as the 'representative length', because tilting results from twisting deformation in the roll bite. Then, the following Eq. is used to judge the occurrence of mode 2 bar tilting, which corresponds to the case without rotational constraint of deformation from the prior stand point.

$$
\bar{M}=\frac{M_{\mathrm{Ent}}}{I_{p} \cdot \bar{\sigma}_{\mathrm{ave}}} \cdot L_{\mathrm{D}}
$$

Here, $L_{D}$ is the maximum projected contact length. Normalized moment $\bar{M}$ is physically the degree of restoration or overturning when a bar can rotate freely. Mode 2 tilting is evaluated on the basis of normalized moment $\bar{M}$.

\section{Experimental Method}

Rolling of a pure lead bar using a hand-cranked mode 1 mill is conducted, and tilting characteristics are measured experimentally. At the entry side of the rolling mill, two guides are installed to control the attitude of the bar before rolling, as is shown in Fig. 6. The first guide fixes the tilt angle before rolling. This guide maintains the tilt angle of the material at $\theta_{\mathrm{s}}$ in section A. Here, $\theta_{\mathrm{s}}$ is defined as the angle between the marking on a pure lead bar before rolling 


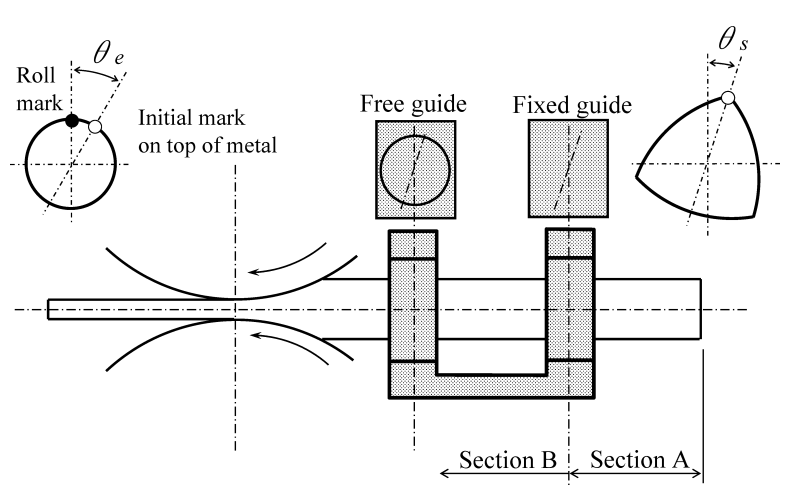

Fig. 6. Structures and positions of guides for experimental caliber rolling.
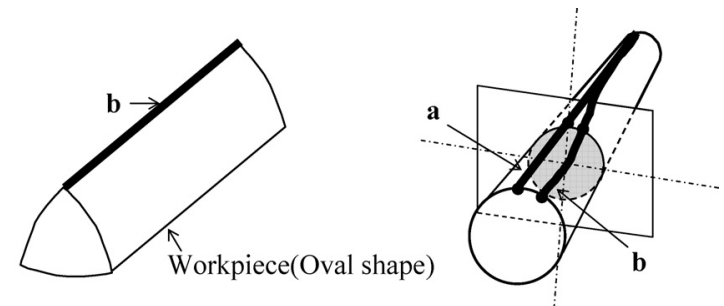

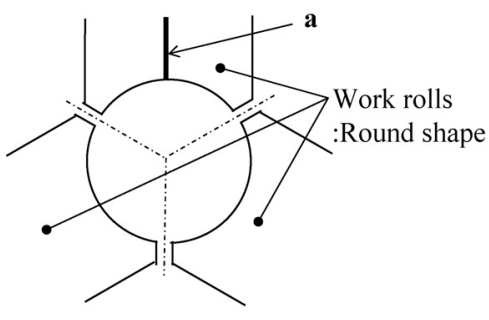

Before rolling

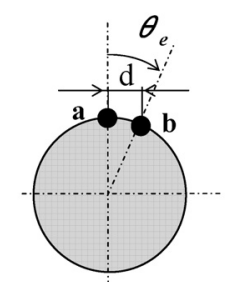

After rolling
Fig. 7. Method of measure of tilt angle.

and the normal position without the overturning. As shown in Fig. 7, the marking line A is drawn at the center of caliber, and this is transcribed in a pure lead rolling.

The second guide allows material to rotate freely in section B. While the end of bar stays within section A, the bar passes the first (fixed) guide. This experiment can reproduce mode 1 tilting, where the tilting of the bar under rolling is represented by twisting deformation in the roll bite. After the bar comes off the first guide, the end of the bar stays in section $\mathrm{B}$, and the bar before rolling can rotate freely. Then, the rolling experiment reproduces mode 2 tilting, which is represented by free rotation of the bar before rolling. After the rolling experiment, by measuring the distance between the position on a lead bar, the tilt angle at the exit, $\theta_{\mathrm{e}}$, is measured along the rolling direction. Here, $\theta_{\mathrm{e}}$ is the angle between the position a (marking of caliber center) and $\mathbf{b}$ (marking of bar before rolling). Tilting is measured as the difference between the tilt angle at the entrance, $\theta_{\mathrm{s}}$, and that at the exit, $\theta_{\mathrm{e}}$.

\section{Results}

\subsection{Analytical Conditions and Experimental Condi- tions}

The types of passes are the hexagon-oval pass, which is of the concave contact type, and the oval-round pass, which is of the convex contact type. Table 1 shows analyti-
Table 1. Analytical conditions.

\begin{tabular}{lc|c}
\hline \hline Pass type & $\begin{array}{c}\text { Oval-Round } \\
\text { Hexagon-Oval }\end{array}$ \\
\hline Roll diameter & $/ \mathrm{mm}$ & 90 \\
\hline Roll speed & $/ \mathrm{rpm}$ & 30 \\
\hline Temperature $/ \mathrm{K}$ & 1273 \\
\hline Flow stress $/ \mathrm{MPa}$ & $\begin{array}{c}\text { Misaka's formula } \\
\bar{\sigma}=128.0 \bar{\varepsilon}^{0.21} \cdot \dot{\bar{\varepsilon}}^{0.13}\end{array}$ \\
\hline Friction coefficient & 0.3 \\
\hline $\begin{array}{l}\text { Front/Back tension } \\
\sigma_{f}, \sigma_{b}\end{array}$ & 0 \\
\hline $\begin{array}{l}\text { Region of analysis } \\
\begin{array}{l}\text { Mesh division } \\
\text { width } \times \text { thickness } \times \text { length }\end{array}\end{array}$ & $\begin{array}{c}\mathrm{N}_{\mathrm{x}} \times \mathrm{N}_{\mathrm{y}} \times \mathrm{N}_{\mathrm{z}} \\
=12 \times 5 \times(2+10+2)\end{array}$ \\
\hline
\end{tabular}

Table 2. Experimental conditions.

\begin{tabular}{lcc}
\hline \hline Pass type & $\begin{array}{c}\text { Oval-Round } \\
\text { Hexagon-Oval }\end{array}$ \\
\hline Roll diameter $\quad / \mathrm{mm}$ & 90 \\
\hline Temperature $\quad / \mathrm{K}$ & Room temperature \\
\hline Material of specimen & Pure lead \\
\hline
\end{tabular}

cal conditions and Table 2 shows experimental conditions. Dimensions of the stock and roll profile are shown in Fig. 8. In the oval-round pass, the effect of the ellipticity of the material, $r_{\mathrm{g}} / y_{0}$, is analyzed. In the experiment, the ellipticity of the material, $r_{\mathrm{g}} / y_{0}$, is only 2.66 . In the hexagonoval pass, the effect of the material shape, $l / A$, is analyzed. The ellipticity of the roll groove, $R_{\mathrm{g}} / Y_{0}$, is constant to keep the reduction constant although the dimensions of the groove are different. In the experiment, $l / A$ are 0.14 and 0.28 .

\subsection{Oval-Round Pass}

The experimental results for the oval-round pass are summarized in Fig. 9. Here, transient change in the tilt angle at the exit, $\theta_{\mathrm{e}}$, is shown for several tilt angles at the entrance, $\theta_{\mathrm{s}}$. When the top of the workpiece enters the roll bite, the end of the bar remains in section A, where the tilt angle before rolling is kept at $\theta_{\mathrm{s}}$ by the fixed guide. The bar under rolling maintains the same situation until the end of the bar comes off the fixed guide. These experimental data lie in the 'section A' interval in Fig. 9. After the end of the bar comes off the fixed guide, it stays in section B where rotation of the bar takes place before rolling. When an inclined bar enters the roll bite, $\theta_{\mathrm{e}}$ exhibits a slight increase in section $\mathrm{A}$, and when the bar enters section $\mathrm{B}, \theta_{\mathrm{e}}$ increases further.

Figure 10(a) shows the relationship between tilt angles at the exit and the entrance in the oval-round pass, obtained by FEM. Since the angle at the entrance is fixed in the analyses, these results correspond to the experimental results of mode 1, where the tilt angle of the bar before rolling are constrained. The values of $\theta_{\mathrm{e}}$ in the experimental results are a little larger than analytical ones. This is considered as follows. The experimental results contain the tilting by the gap between guide and bar. Moreover, it includes the tilting between entry position of roll bite and fixed guide. So, absolute values of experiments are larger than that of analyses. 


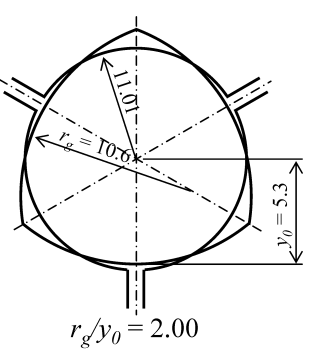

(a) Oval-round pass
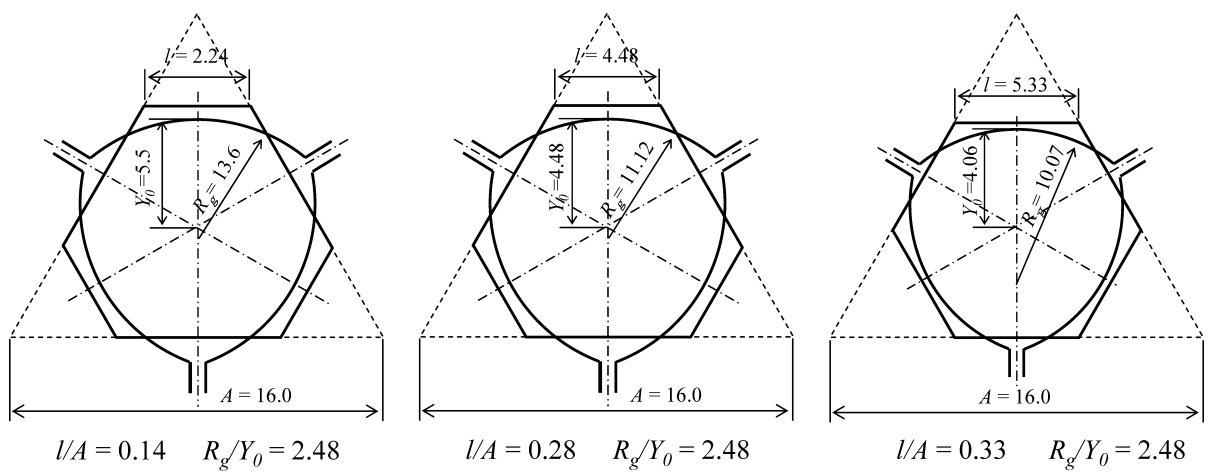

(b) Hexagon-oval pass

Fig. 8. Dimension of stock and roll profile.

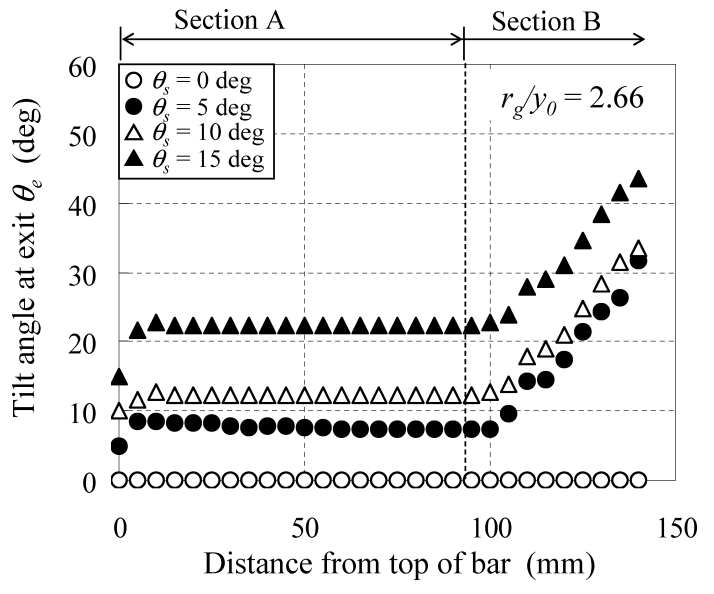

Fig. 9. Transient change in tilt angle at exit $\theta_{\mathrm{s}}$ in oval-round pass, obtained by experiment.

Analytical results indicate that the bar tends to overturn for any $\theta_{\mathrm{s}}$, similar to the experiment results. Figure $10(\mathrm{~b})$ summarizes the analytical results of normalized moment $\bar{M}$ which acts at the entrance of the deformation zone in the oval-round pass. Normalized moment $\bar{M}$ is comparable to the experimental results (mode 2) for Section B, where the bar before rolling can rotate freely. Normalized moment $\bar{M}$ shows that the bar tilt angle at exit line will increase for any $\theta_{\mathrm{s}}$, similar to the experiment results. The analytical results for mode 1 and mode 2 show that the effect of the ellipticity of the material is very small. In the oval-round pass, it was found that the bar tilt angle at exit was difficult to decrease in the roll bite once tilting occurred.

\subsection{Hexagon-Oval Pass}

The experimental results for the hexagon-oval pass are summarized in Fig. 11. When an inclined bar enters the roll bite, $\theta_{\mathrm{e}}$ exhibits a slight increase in section A. When the bar enters section $\mathrm{B}$, the bar tends to overturn for any $\theta_{\mathrm{s}}$ in the case of $l / A=0.14$. On the other hand, if $\theta_{\mathrm{s}}$ is smaller than 20 degrees in the case of $l / A=0.28$, the bar tends to be gradually restored. In a word, it can be said that phenomenon of restoration becomes easier to occur in section $\mathrm{B}$ when $l / A$ becomes longer.

Figure 12(a) shows the relationship between the tilt angle at the exit and that at the entrance in the hexagon-oval pass, obtained by FEM. The bar tends to overturn for any $\theta_{\mathrm{s}}$. The predictions obtained by FEM are in good agreement with the measurements. Figure 12(b) summarizes the analytical results of normalized moment $\bar{M}$ which acts at the entrance of the deformation zone in the hexagon-oval pass. As in the case of $l / A=0.14$ and 0.28 , normalized moment $\bar{M}$ values are negative, the bar tilt angles are predicted to increase in the roll bite. However, as in the case of $l / A=0.33$, normalized moment $\bar{M}$ values are positive, the bar will be restored. In mode 2 , restoration of the rolled bar is easy to occur when $l / A$ is large. It is considered that the analytical results of mode 2 are not quantitatively corresponding to the experimental results, but the $\bar{M}$ change tendency grows are qualitatively corresponding to those.

\subsection{Bar Attitude under 3-Roll Rolling}

From the experimental results of Fig. 9 and Fig. 11, the bar tends to overturn for both cases of Oval-Round (the convex contact type) and Hexagonal-Oval (the concave contact type). When the results of Fig. 10 compare with that of Fig. 12, the tendency of attitude of bar is the same. This is considered as follows. In the case of Hexagonal-Oval rolling, the metal of bar mainly flows in the radial direction from surface to center (rolling pressure direction). This tendency is the same in rolling of Oval-Round. In a word, metal of bar in 3-roll rolling hardly flows to the direction of 


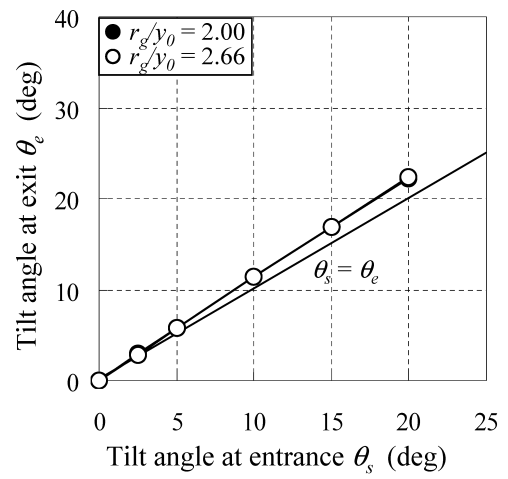

(a) Mode 1 tilting

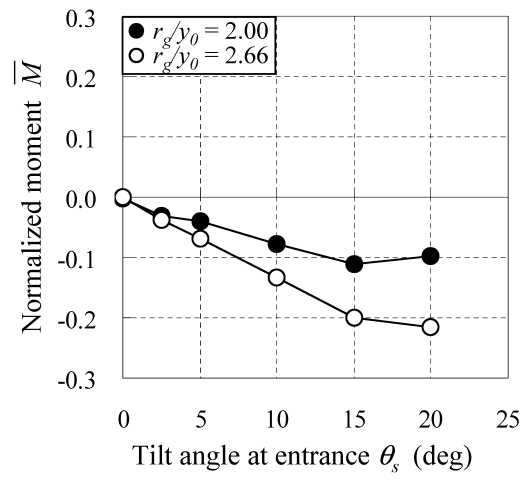

(b) Mode 2 tilting

Fig. 10. Tilt angle at exit $\theta_{\mathrm{e}}$ and normalized moment $\bar{M}$ in oval-round pass, obtained by FEM.
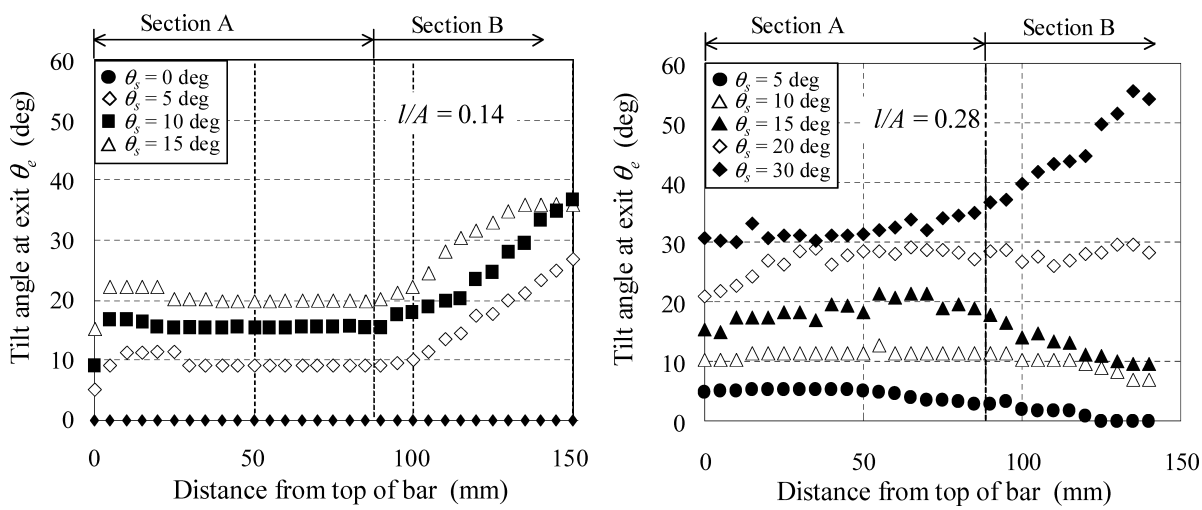

Fig. 11. Transient change in tilt angle at exit $\theta_{\mathrm{s}}$ in hexagon-oval pass, obtained by experiment.

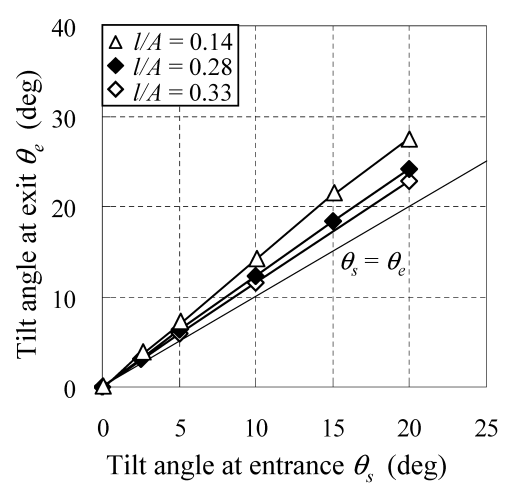

(a) Mode 1 tilting

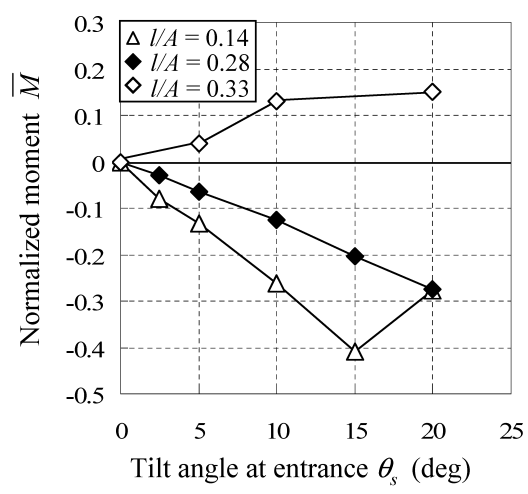

(b) Mode 2 tilting

Fig. 12. Tilt angle at exit $\theta_{\mathrm{e}}$ and normalized moment $\bar{M}$ in hexagon-oval pass, obtained by FEM.

circumference, so any caliber condition shows the attitude being scarcely overturned.

This is considered as follows. In the case of HexagonalOval rolling, the inclination angle of Oval caliber wall is small like a flat rolling, and the metal is received strong force to rolling pressure direction. Therefore, the deformation of tilting by overturning or restoration is not caused easily under rolling. As the metal hardly flows in the direction of width, the force which rules the overturning or the restoration faces the rolling pressure direction, and rolled metal bar slightly shows the overturning in the case of mode 1 under Hexagonal-Oval rolling.

In the case of Round rolling, the Round caliber wall faces the center direction of caliber, and the metal is received strong force to the center direction of caliber. As the metal mainly flows in the direction of center, the rolled metal bars slightly show the overturning. In a word, metal of bar in the both case of Oval and Round caliber in 3-roll rolling hardly flows to the direction of circumference, so it is considered that any caliber condition shows the attitude being scarcely overturned.

\section{Conclusion}

Tilting under 3-roll rolling was examined by FE analysis and using a model experiment. The modes of tilting in the oval-round pass and hexagon-oval pass were clarified by comparing the experimental results and the numerical results. The following conclusions were drawn from the results of this work. 
(1) A method of analyzing tilting under 3-roll rolling by steady-state FE analysis was devised. The predicted results agreed well with the experimental measurements for mode 1 tilting. If it is the case of similar caliber shape, the tendency of tilting of Mode 2 can be qualitatively estimated by this analytical method. It can be concluded that FE analysis is helpful for the design of calibers and rolling conditions of bar rolling and rod rolling.

(2) The mechanism of tilting differs depending on the contact contour. In the oval-round pass, which is of the convex contact type, the both cases of mode 1 and mode 2 tend to overturn and never show restoring, that is, the bar tilt angle was difficult to decrease in the roll bite once tilting occurred. In the hexagon-oval pass, which is of the concave contact type, tilting phenomena occur in all the case of mode 1 . But $l / A$ becomes larger, the bar tilt angle tends to decrease in the roll bite. In short, although the bar tends to overturn when the entrance cross section is constrained strongly, the bar tilt angle is expected to decrease when the constraint becomes relatively small.

\section{REFERENCES}

1) T. Ohno: Tetsu-to-Hagané, 55 (1969), 1286.

2) M. Shternov: Stahl, (1963), No. 11, 874.

3) M. Asakawa: The Proc. of the 1970 Japanese Spring Conf. for the Technology of Plasticity, (1970), 141.

4) M. Asakawa, H. Miyazawa, M. Toi, Y. Katayama and J. Yanagimoto: Tetsu-to-Hagané, 89 (2003), No. 7, 758.

5) M. Asakawa, M. Toi, Y. Katayama, K. Kobayashi and J. Yanagimoto: Proc. of NUMIFORM 2004, AID, Melville, NY, (2004), 463.

6) S. Mroz, A. Milenin and H. Dyja: Metal Forming 2004, (2004), 97.

7) J. Yanagimoto, M. Kiuchi, M. Nakamura and R. Kurahashi: J. Jpn. Soc. Technol. Plast., 32, (1991), 1000.

8) J. Yanagimoto: Seisan-Kenkyu, 43 (1991), No. 11, 512.

9) J. Yanagimoto: Seisan-Kenkyu, 49 (1997), No. 9, 414.

10) T. Sasaki, J. Yanagimoto, T. Kouno and M. Kiuchi: Tetsu-to-Hagané, 79 (1993), No. 3, 360.

11) T. Udagawa, Y. Takashima, M. Yoshida and J. Yanagimoto: Proc. of the Steel Rolling 98, ISIJ, Tokyo, (1998), 359.

12) J. Yanagimoto: J. Mater. Process. Technol., 130 (2002), 224.

13) J. Yanagimoto, M. Kiuchi, H. Miyazawa and M. Asakawa: Tetsu-toHagané, 86 (2000), No. 7, 452.

14) J. Yanagimoto, Y. Kadomura, T. Muto and K. Inoue: Steel Res., 73 (2002), No. 12, 526. 